\title{
ARTÍCULO ORIGINAL \\ Caracterización del módulo salud del sistema 123: atención de accidentes de tránsito en la ciudad de Medellín
}

\author{
Characterization of the health module of the 123 system for \\ traffic accident attention in the city of Medellín / Caracterização \\ do módulo saúde do sistema 123: atenção de acidentes de \\ trânsito na cidade de Medellín
}

Cindy Zuluaga Ramírez ${ }^{1}$, Ana Cortés Ballesteros ${ }^{1}$, Sebastián Medina Rivera ${ }^{1}$, Juan Duque Pérez $^{1}$, Santiago Upegui Estrada ${ }^{1}$, Mateo Ochoa Franco ${ }^{1}$, Jorge Cohen Cajiao ${ }^{1}$, Diego Abreo Leal ${ }^{2}$

\section{RESUMEN}

Objetivo: Caracterizar la atención de accidentes de tránsito en la ciudad de Medellín para obtener una imagen general de la situación de salud en este contexto.

Metodología: Estudio descriptivo observacional, con intención analítica, de fuentes secundarias de información, en pacientes con edad mayor o igual a 16 años, atendidos por el personal 123, módulo salud, víctimas de accidentes de tránsito en Medellín.

Resultados: Un total de 3829 individuos sufrieron accidente de tránsito entre octubre y diciembre del 2018 según los criterios del estudio. De estos, el 63.1\% de sexo masculino, con edad media de 34 años. La zona con mayor demanda de atención prehospitalaria fue la comuna 10 de la ciudad (La Candelaria) y la mayoría de los afectados por los accidentes lo fueron en calidad de conductor, $64 \%$, y en un $75 \%$ de los casos, el vehículo involucrado fue una motocicleta. Solo el $26.5 \%$ de los pacientes fueron dados de alta en la escena. Las extremidades fueron las zonas corporales de mayor compromiso, en un $78.2 \%$ de los episodios, seguidas por el cráneo y la cara, con un $26.8 \%$.

Conclusiones: Es evidente la necesidad de la ciudad de implementar y evaluar el impacto de estrategias dinámicas que permitan un manejo eficiente de los recursos, que priorice las zonas y grupos demográficos de mayor riesgo y la articulación de la disposición de pacientes dentro de la cadena de atención, según los tipos más frecuentes de lesiones. Palabras clave: accidentes de tránsito; extremidades; traumatismos craneocerebrales; atención prehospitalaria

\section{ABSTRACT}

Objective: To characterize the traffic accident attention in the city of Medellín in order to obtain a general image of the health situation in this context.

Methodology: Observational descriptive study, with analytic intent, of secondary information sources in patients with age higher or equal to 16 years of age, served by the 123 personnel, health module, who were victims of traffic accidents in Medellín.

Results: A total of 3829 individuals suffered traffic accidents between October and December of 2018 according to the study criteria. Of these, $63.1 \%$ were males, with an average age of 34 years old. The zone with the highest demand of pre-hospital attention was the Comuna 10 of the city (known as La Candelaria) and most of the affected by the accidents were as drivers, $64 \%$, and $75 \%$ of the cases, the vehicle involved was a motorcycle. Only $26.5 \%$ of the patients were discharged on the scene. The extremities were the body zones with the highest compromise in $78.2 \%$ of the episodes, followed by the cranium and the face, with $26.8 \%$.
Fecha de recibido: 19 de febrero de 2020 Fecha de aprobación: 29 de abril de 2020

Forma de citar este artículo: Zuluaga C, Cortés A, Medina S, Duque J, Upegui S, Ochoa M, et al. Caracterización del módulo salud del sistema 123: atención de accidentes de tránsito en la ciudad de Medellín. Med UPB. 2020;39(2):11-16. DOI:10.18566/medupb.v39n2.a03

\footnotetext{
1 Universidad CES. Medellín, Colombia.

2 Hospital Pablo Tobón Uribe. Medellín, Colombia.
}

Dirección de correspondencia: Cindy Zuluaga Ramírez. Correo electrónico: cindyzeta33@ outlook.es 
Conclusions: It is evident that the city needs to implement and evaluate the impact of dynamic strategies that enable an efficient management of the resources that prioritizes the zones and demographic groups with the highest risk and the articulation of the disposition of patients within the attention chain, according to the most frequent types of injuries.

Keywords: accidents, traffic; extremities; craniocerebral trauma; prehospital care

\section{RESUMO}

Objetivo: Caracterizar a atenção de acidentes de trânsito na cidade de Medellín para obter uma imagem geral da situação de saúde neste contexto.

Metodologia: Estudo descritivo observacional, com intenção analítica, de fontes secundárias de informação, em pacientes com idade maior ou igual a 16 anos, atendidos pelo pessoal 123, módulo saúde, vítimas de acidentes de trânsito em Medellín.

Resultados: Um total de 3829 indivíduos sofreram acidente de trânsito entre outubro e dezembro de 2018 segundo os critérios do estudo. Destes, 63.1\% de sexo masculino, com idade média de 34 anos. A região com maior demanda de atenção pré-hospitalar foi a comuna 10 da cidade (La Candelaria) e a maioria dos afetados pelos acidentes foram em qualidade de condutor, $64 \%$, e num $75 \%$ dos casos, o veículo envolvido foi uma motocicleta. Só $26.5 \%$ dos pacientes foram dados de alta na cena. As extremidades foram as zonas corporais de maior compromisso, em $78.2 \%$ dos episódios, seguidas pelo crâneo e a cara, com um $26.8 \%$.

Conclusões: É evidente a necessidade da cidade de implementar e avaliar o impacto de estratégias dinâmicas que permitam um manejo eficiente dos recursos, que priorize as zonas e grupos demográficos de maior risco e a articulação da disposição de pacientes dentro da cadeia de atenção, segundo os tipos mais frequentes de lesões.

Palavras chave: acidentes de trânsito; extremidades; traumatismos crâneo-cerebrais; atenção pré-hospitalar

\section{INTRODUCCIÓN}

Los accidentes de tránsito son una de las principales causas de lesiones en la población económicamente productiva, por lo que implementar estrategias para el contacto oportuno con estos pacientes, desde el contexto prehospitalario, es importante para mejorar el pronóstico y reducir las tasas de discapacidad ${ }^{1}$.

El manejo eficiente de un evento vital involucra triage, tratamiento y transporte. Las herramientas de clasificación predicen qué pacientes tienen mayor probabilidad de sobrevivir, de morir o necesidad de medidas avanzadas e invasivas de soporte, principio que rige el concepto de direccionamiento pertinente, de acuerdo con las necesidades de cada paciente. En este proceso hay que tener en cuenta, también, la disponibilidad de recursos necesarios para la rehabilitación y seguimiento ${ }^{2}$.
$\mathrm{Al}$ revisar la literatura, se encuentra que los sistemas de atención prehospitalaria en países escandinavos (centros con mayor experticia), tienen un impacto positivo sobre el pronóstico a corto y mediano plazo, con tasas de reducción de mortalidad de hasta un $17 \%$ en víctimas de traumas graves, que pueden ser atendidos oportunamente en centros de alta complejidad, lo que requiere articulación entre el medio prehospitalario y el escenario hospitalario, ya que permite que se comporten como un ente único, al prestar una atención oportuna y al direccionar correctamente a los pacientes ${ }^{3}$.

Con estos antecedentes planteamos la necesidad de caracterizar el comportamiento del servicio de atención prehospitalaria en Medellín, con respecto a los accidentes de tránsito, para retratar una imagen general de la situación de salud en este contexto y proponer una base para generar estrategias que mejoren la atención $\mathrm{y}$ direccionamiento de los pacientes. 


\section{METODOLOGÍA}

Este es un estudio descriptivo de tipo observacional, con intención analítica, que cuenta con disponibilidad retrospectiva de datos (fuente secundaria de información). Realizado en pacientes con edad mayor o igual a 16 años, atendidos por el personal de la línea de emergencias 123, módulo salud - víctimas de accidentes de tránsito, en la ciudad. El periodo de observación fue el último trimestre de 2018.

Los análisis estadísticos fueron ejecutados en IBM SPSS Statistics 22, Stata versión 16, y los mapas fueron creados en AcrGIS for Desktop, versión 10.7.1. Se excluyeron los pacientes con llamadas por causa de una patología médica, mujeres que, por registro se encontraban en estado de embarazo, víctimas de abuso sexual, personas encontradas por personal de emergencia sin notificación al módulo 123 y, en general, quienes tuvieran un motivo de atención diferente a urgencia traumática.

\section{RESULTADOS}

Fueron identificados 4761 pacientes, se excluyeron 928 que no cumplían criterios de inclusión; por lo que quedaron 3829 casos de accidente de tránsito entre octubre y diciembre del 2018.

El 63,1\% de casos fue de individuos de sexo masculino. La edad media fue 34 años, el 67\%, en el intervalo entre 20 y 40 (Tabla 1 ).

Tabla 1. Características sociodemográficas de 3829 pacientes atendidos por el módulo 123, por accidentes de tránsito - último trimestre de 2018 en Medellín.

\begin{tabular}{llc}
\hline Característica & \multicolumn{1}{c}{ Categoría } & $\begin{array}{c}\text { Frecuencia } \\
\text { en porcentaje }\end{array}$ \\
\hline \multirow{2}{*}{ Sexo } & Femenino & 36.9 \\
& Masculino & 63.1 \\
\hline \multirow{3}{*}{ Edad } & $40-60$ años & 17.7 \\
& $20-40$ años & 67.1 \\
& Menos de 20 años & 8.3 \\
& Más de 60 años & 6.8 \\
\hline
\end{tabular}

La zona de la ciudad donde hubo mayor demanda de atención prehospitalaria fue la comuna 10 (La Candelaria), con más incidencia de emergencias los miércoles y sábados, y mayor ocurrencia en horas de la tarde, entre la 1 p.m., y 6 p.m.

La mayoría de los accidentes sucedieron a conductores, con un 64\%. En el 75\% de los casos, el vehículo involucrado fue una motocicleta, con una cinemática, en la mayoría de los casos, de baja energía, en un 63.8\%.

La clasificación en el triage fue de color amarillo, en la mayoría de los casos, lo que corresponde a un $65 \%$. La clasificación en la escena fue triage III, en un $61.7 \%$. E1 73,5\% de los pacientes continuaron en el proceso de atención y fueron trasladados a una institución prestadora de salud (IPS), mientras que el $26.5 \%$ de los pacientes, tras la exclusión de condiciones de alto riesgo, fueron dados de alta en la escena.

Las extremidades comprenden las zonas corporales de mayor compromiso en los accidentes, con un resultado del 78,2\%, seguidas por el cráneo y la cara (26.8\%). Entre las regiones anatómicas con menos lesiones esta la pelvis (1\%). Las demás partes del cuerpo se muestran en la Tabla 2. En lo atinente a las características clínicas según los signos vitales, el hallazgo inicial más frecuente fue la taquicardia (32.3\%) y la taquipnea (19.1\%), la hipotensión solo se presentó en el $0.6 \%$ de los pacientes. La mortalidad en la escena fue del $0.05 \%$.

Tabla 2. Zonas corporales afectadas en 3829 accidentes de tránsito ocurridos durante el último trimestre de 2018 en la ciudad de Medellín.

\begin{tabular}{lc}
\hline \multicolumn{1}{c}{ Zona anatómica } & Porcentaje \\
\hline Extremidades & 78.2 \\
Cráneo-encéfalo & 26.8 \\
Tórax & 12.9 \\
Otras zonas & 9 \\
Cadera & 5.7 \\
Abdomen & 4.4 \\
Cuello & 3.5 \\
Pelvis & 1 \\
\hline
\end{tabular}

La mayor frecuencia de atención prehospitalaria se registró en la comuna 10 ( $\mathrm{La}$ Candelaria) y en la comuna 8 (Villa Hermosa). Las razones de ocurrencia, y los detalles geográficos en la ciudad se pueden apreciar en la Figura 1.

En el 64\% de las atenciones el tiempo entre la activación y el despacho del servicio está entre uno y cinco minutos. En la Figura 2 se pueden ver los intervalos de tiempo según las comunas.

El tiempo entre el despacho de la ambulancia hasta la llegada a la escena fue de 5 a 15 minutos en el $62 \%$ de los casos, y desde la atención en la escena hasta la disposición final del paciente en un centro hospitalario, fue de entre 60 a 90 minutos en el $27.8 \%$ de los episodios. La Figura 3 muestra el tiempo transcurrido desde el despacho hasta 
la llegada de la ambulancia al lugar del accidente, y en la Figura 4 se puede ver el tiempo desde la activación del traslado hasta la llegada a la IPS.

En el caso de la comuna 7 (Robledo) se observaron extensos intervalos de tiempo totales desde la activación del sistema hasta el despacho de la ambulancia, y entre la activación del sistema para el traslado hasta la entrega del paciente a la institución de destino. El mayor intervalo de tiempo entre el despacho de la ambulancia hasta la llegada a la escena fue para la comuna 14 (El Poblado).

\section{DISCUSIÓN}

El presente estudio comprende la caracterización del registro de atención prehospitalaria de trauma en

Figura 1. Razón de ocurrencia de eventos por el número correspondiente a cada comuna de la ciudad de Medellín.

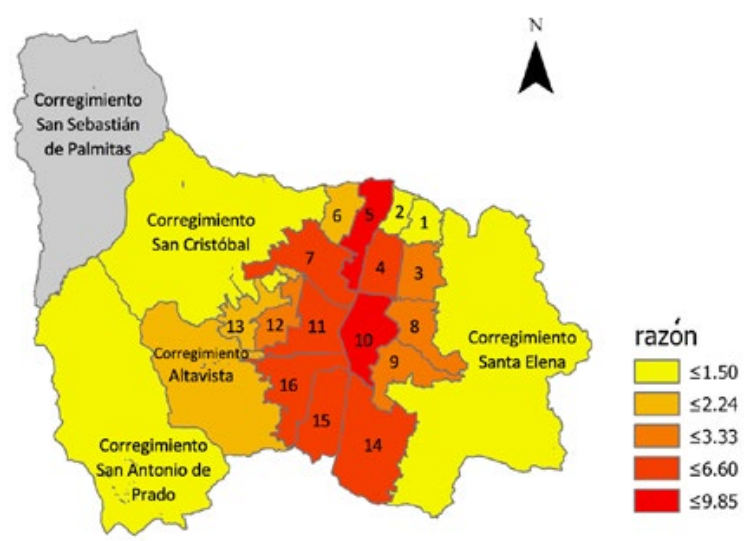

Figura 3. Intervalo de tiempo en minutos, desde despacho de la ambulancia hasta la llegada a la escena.

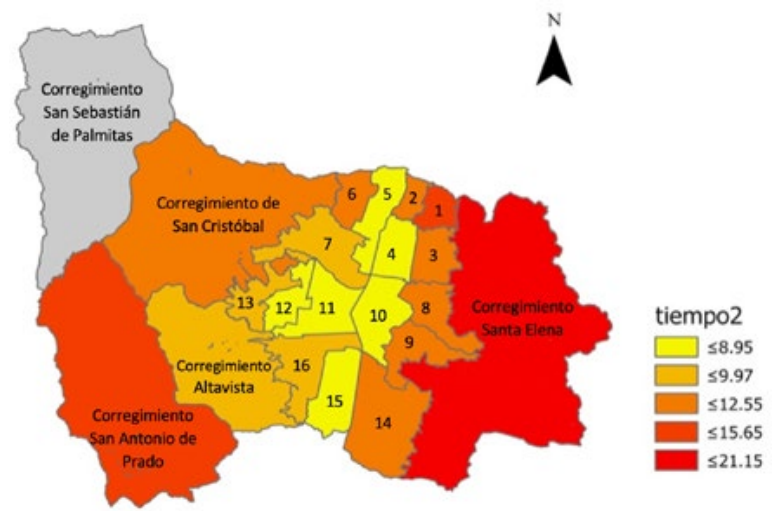

Medellín durante el último trimestre del año 2018. Los hallazgos son coherentes con lo descrito en la literatura médica, es claro que la supervivencia y el impacto sobre la morbilidad en trauma dependen del tiempo, de la identificación temprana, intervención y traslado oportuno de pacientes a instituciones médicas con capacidad de atención coherente con estas necesidades ${ }^{2}$.

Lo anterior justifica la conformación de equipos de atención prehospitalaria para el manejo oportuno, integral y pertinente de los pacientes con trauma en los diferentes escenarios ${ }^{4}$. Medellín cuenta con 13 ambulancias, de las cuales 12 son básicas, y una medicalizada, mientras que Bogotá, la capital del país, tiene 78 ambulancias básicas y 21 medicalizadas 5 .

Medellín tiene 2901948 habitantes (datos del censo nacional para 2019), ubicados en 382 kilómetros cua-

Figura 2. Intervalo de tiempo en minutos, entre la activación del sistema de atención hasta el despacho de la ambulancia.

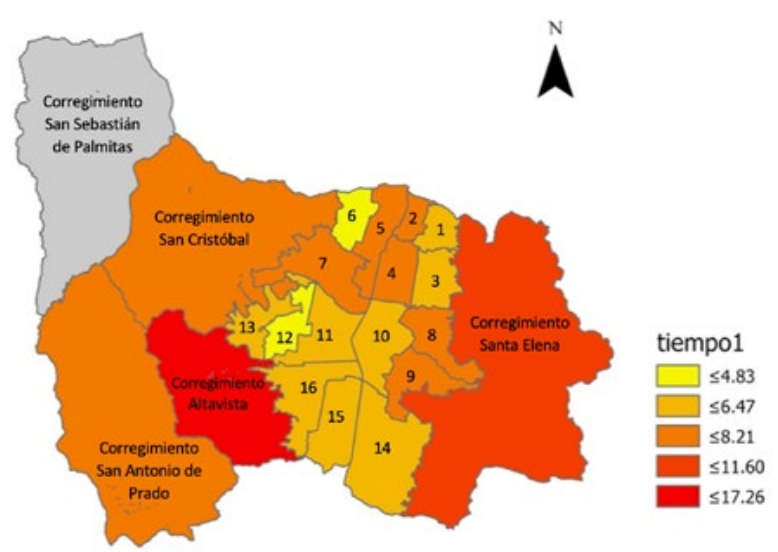

Figura 4. Intervalo de tiempo en minutos desde la activación del traslado del paciente hasta la llegada a la institución prestadora de salud.

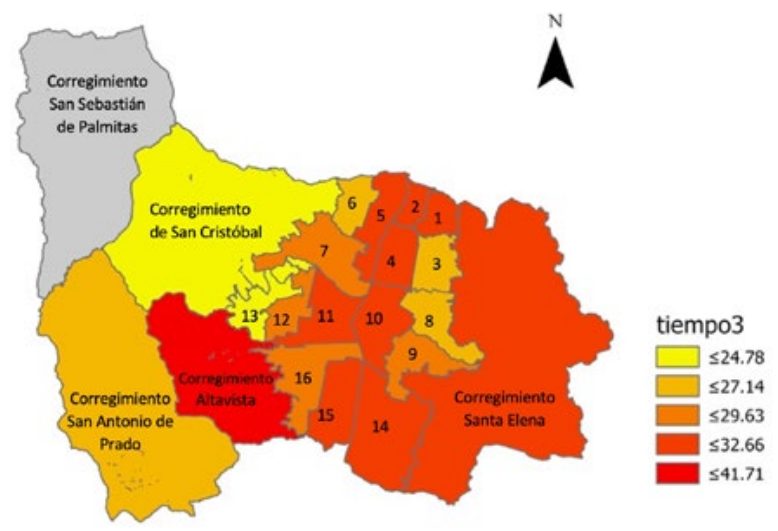


drados, donde, según el Registro Único Nacional de Tránsito (RUNT), se estima la presencia y circulación de 23444 automóviles (acumulado para agosto del 2017) ${ }^{6,7}$, esto, sin tener en cuenta a las motocicletas. La tasa de mortalidad por accidentes de tránsito ha descendido gradualmente durante los últimos 5 años, con una tasa de mortalidad estimada, para el 2018, de 9.4 por cada 100 000 habitantes, donde el $47.1 \%$ de las fatalidades ocurren en peatones ${ }^{6,7,8}$. Estas cifras evidencian la importancia de registrar e investigar sobre el comportamiento de este problema, que no ha sido documentado (según la revisión de bases de datos como Pubmed, Google Scholar y otras fuentes de literatura gris).

$\mathrm{E}$ l análisis de datos de las bitácoras de atención del 123 identifica que la mayoría de las víctimas de accidentes de tránsito son hombres en edad productiva, la gran mayoría en calidad de conductor de motocicleta, lo que concuerda con registros internacionales. Un hallazgo de importancia fue la relativamente baja frecuencia de trauma encefálico, del 26.8\%, comparada con los datos del registro alemán de trauma para el año de 2017, que gira en torno al $47.1 \%$ en ese país. Contrasta el alto porcentaje de lesiones en extremidades, próximo al 80\%, que en el registro alemán representan alrededor de la mitad de los casos, lo que plantea que cada lugar tiene necesidades específicas, tanto en implementos para la dotación de las ambulancias, como en el entrenamiento del personal que atiende a los pacientes (en el caso de las lesiones de extremidades, para identificar lesiones vasculares, inmovilizar y realizar una alineación inicial del miembro afectado) ${ }^{9}$.

Existe un registro panamericano de trauma, que incluye víctimas de accidentes de Colombia, específicamente la ciudad de Cali, donde se encuentran pacientes con características sociodemográficas similares a Medellín, aun cuando el registro no es exclusivo para trauma por accidente de tránsito. En este, se refleja una mortalidad que va del 0.1 al 37\%, sin discriminar fatalidades en escena, que en nuestro estudio corresponden al $0.05 \%$. La cifra reportada en el presente estudio es menor que la de otros que alcanzan hasta el 3.6\%. Entre las explicaciones plausibles de la discrepancia se cuenta la atención prehospitalaria prestada. En todos los estudios se visualiza que se debe prestar especial atención, en escenario de trauma, a las estrategias de salud pública dirigidas a personas en edad productiva ${ }^{10,11}$.

Tanto en Medellín como en Bogotá se adelantan estrategias para optimizar la atención prehospitalaria, como la ubicación de ambulancias por georreferenciación en zonas de mayor accidentalidad y grupos poblacionales expuestos. Lo anterior ha tenido un impacto positivo sobre los tiempos de respuesta. Con esta estrategia se ha logrado que en La Candelaria y Castilla (con importante índice de accidentalidad), el tiempo entre la activación del sistema y el despacho de la ambulancia sea menor a cinco minutos, y un intervalo de tiempo entre el despacho de la ambulancia y la llegada a la escena, entre 5 y 15 minutos. De todas formas, otras comunas de la ciudad están en el mismo rango temporal. Hay diferentes puntos de corte de los tiempos para mejorar la supervivencia. Cuando se tarda ocho minutos prácticamente no hay mortalidad debida al tiempo de atención, y esta llega solo al $0.1 \%$ cuando se tardan 17 minutos (3.1\% después de este último límite). Según lo anterior, el tiempo hasta la llegada a la escena de la ambulancia en Medellín podría tener que ver con la baja mortalidad, lo que deberá ser verificado en estudios analíticos $5,12,13,14$.

Este estudio es un punto de partida para construir estrategias basadas en el diagnóstico de la situación, que permitan optimizar la atención y direccionamiento oportunos de estos pacientes, lo que repercutiría positivamente sobre los tiempos de estancia hospitalaria, la pérdida de funcionalidad e incluso sobre la posibilidad de muerte, así como en las consecuencias económicas del problema ${ }^{13,14}$.

Otro punto importante para resaltar tiene que ver con ubicación de los pacientes, que debe tener en cuenta las necesidades de salud identificadas durante la atención prehospitalaria. La mayoría de las víctimas de accidente de tránsito sufren trauma con cinemática de baja energía y clasificación amarilla o III, lo que implica derivarlos a instituciones de mediana complejidad. Sin embargo, muchos pacientes son trasladados a instituciones de alta complejidad. Por lo anterior, hacen falta campañas de formación, que permitan consolidar el sistema de remisión para lograr un uso más pertinente de recursos ${ }^{15,16}$.

Para concluir, la información obtenida muestra diferencias respecto a otros países y refleja las necesidades de la ciudad en cuanto a estrategias que permitan un manejo eficiente de los recursos. En dichas estrategias se deben tener en cuenta las áreas de mayor necesidad en el abordaje, manejo y disposición del paciente dentro de una cadena de atención.

\section{DECLARACIÓN DE CONFLICTO DE INTERESES}

Los autores declaran no tener ningún conflicto de intereses.

Agradecimientos especiales a la Dra. Marie Claire Berrouet por su acompañamiento, y al módulo 123 por autorizar el acceso a las bases de datos de atención a los investigadores. 


\section{REFERENCIAS}

1. Mower-Wade D, Bartley MK, Pirrung J. Trauma care. Nursing. 2005; 35: doi:10.1097/00152193200505002-00004

2. Purtill MA, Benedict $K$, Hernández-Boussard $T$, et al. Validation of a prehospital trauma triage tool: A 10-year perspective. J Trauma. 2008; 65(6):1253-7.

3. Murad MK, Larsen $\mathrm{S}$, Husum H. Prehospital trauma care reduces mortality. Ten-year results from a time-cohort and trauma audit study in Iraq. Scand J Trauma Resusc Emerg Med. 2012; 20:1-10.

4. Mackersie R. History of trauma field triage development and the American College of Surgeons criteria. Prehospital Emerg Care. 2006; 10(3):287-94.

5. Octaviano J, Gutiérrez B [Internet]. Cantidad y distribución de ambulancias para responder con oportunidad y eficacia a los incidentes médicos y de atención pre hospitalaria que se suscitan en la ciudad de Bogotá durante el año 2018 [citado el 18 de febrero de 2020]. Disponible en: https://repository.javeriana.edu.co/handle/10554/40657

6. Andi [Internet]. Informe del sector automotor [citado el 27 de enero de 2020]. Disponible en: http://www.andi.com.co/Uploads/8

7. Alcaldía Medellín [Internet]. Medellín y su población. Documento técnico de soporte POT (Acuerdo 46/2006) [citado el 27 de enero de 2020]. Disponible en: http://www.medellin.gov. co/irj/go/km/docs/wpccontent/Sites/Subportal del Ciudadano/Plan de Desarrollo/Secciones/ Informaci?n General/Documentos/POT/medellinPoblacion.pdf

8. Secretaría de Movilidad de Medellín [Internet]. Indicadores - Secretaría de Movilidad de Medellín [citado el 27 de enero de 2020]. Disponible en: https://www.medellin.gov.co/movilidad/m-enlinea/indicadores\#4-incidentes-viales

9. Society GT. German trauma registry, annual report 2017. Sekt NIS Ger Trauma Soc. 2017; 21(5):430.

10. Ordóñez CA, Morales M, Rojas-Mirquez JC. Trauma registry of the Pan-American Trauma Society: One year of experience in two hospitals in southwest Colombia. Colomb Medica. 2016; 47(3):148-54.

11. Pham $H$, Puckett $Y$, Dissanaike $S$. Faster on-scene times associated with decreased mortality in Helicopter Emergency Medical Services (HEMS) transported trauma patients. 2017;2:1-5.

12. Velásquez Osorio N, Naranjo Luján S. Indicadores de atención pre hospitalaria para incidentes viales en Colmbia. RevFacNaSalud Pública. 2017; 35(3):410-9.

13. Pons PT, Markovchick VJ. Eight minutes or less: does the ambulance response time guideline impact trauma patient outcome? J Emerg Med. 2002; 23(1):43-8.

14. Daniels B, Venkatesh A. Increased computed tomography scan use among emergency department patients with abdominal pain does not increase diagnostic certainty. Ann Emerg Med. 2014; 64(4):S23.

15. Tudela P, Mòdol JM. La saturación en los servicios de urgencias hospitalarios. Emergencias. 2015; 27(2):113-20.

16. Restrepo-Zea JH, Jaén-Posada JS, Espinal JJ, Zapata PA. Saturación en los servicios de urgencias: análisis de cuatro hospitales de Medellín y simulación de estrategias. Rev Gerenc Polít Salud. 2018; 17(34):1-15. 